(C) 2021 Universidad Nacional Autónoma de México, Facultad de Estudios Superiores Zaragoza.

Este es un artículo Open Access bajo la licencia CC BY-NC-ND (http://creativecommons.org/licenses/by-nc-nd/4.0/).

TIP Revista Especializada en Ciencias Químico-Biológicas, 24: 1-12, 2021.

https://doi.org/10.22201/fesz.23958723e.2021.332

\title{
Desregulación metabólica y consecuencias clínicas por el consumo de fructosa
}

\author{
María Guadalupe Alcántara-Ortiz ${ }^{1}$, \\ Jesús Campos-Serrano ${ }^{1,2}$ y Alfredo Ibarra-Sánchez ${ }^{1,3 *}$ \\ ${ }^{1}$ Universidad Insurgentes. Plantel Viaducto-Coruña, Ciudad de México, 03510, México. ${ }^{2}$ Departamento \\ de Biología, Universidad Autónoma Metropolitana, Unidad Iztapalapa, Ciudad de México, 09340, \\ México. ${ }^{3}$ Departamento de Farmacobiología, Centro de Investigación y de Estudios Avanzados del IPN. \\ Unidad Sur, Alcaldía Tlalpan, Ciudad de México, 14330, México. E-mail: *aibarra@cinvestav.mx
}

\begin{abstract}
RESUMEN
La industria alimentaria ha incrementado su tasa de producción por alimentos siempre disponibles, listos para su consumo o que requieren de una mínima preparación, pero sin que aumente su calidad y sólo para satisfacer la demanda de una sociedad moderna. La característica que tienen en común estos alimentos, es el uso de un producto derivado del almidón del maíz denominado Jarabe de Maíz con un alto contenido de Fructosa (JMAF), el almidón es ampliamente usado en la fabricación de este tipo de alimentos procesados, y cuyo consumo desmedido está asociado con el incremento en la tasa de obesidad y de trastornos metabólicos.

El metabolismo de la fructosa, conlleva a la disminución de la cantidad de energía disponible en el organismo. Simultáneamente, la producción de metabolitos ocasiona cambios en las vías de señalización celular promoviendo un incremento en la cantidad de lípidos como los triglicéridos y el colesterol de muy baja densidad. Los altos niveles de estas moléculas favorecen condiciones que encaminan a la obesidad y a la resistencia a la insulina, propiciando el desarrollo de la diabetes mellitus tipo 2, hiperuricemia e inflamación que por lo general son la base para el origen del cáncer y el inicio de las enfermedades neurológicas. Esta revisión se centra en la relación entre la fructosa y estas enfermedades y su posible mecanismo de acción.
\end{abstract}

Palabras clave: fructosa, hiperuricemia, diabetes mellitus tipo 2, lipogénesis, inflamación.

\section{Metabolic dysregulation and clinical consequences of fructose consumption}

\begin{abstract}
In order to meet modern societies' demand for always-available instant or ready-to-eat foods, the food industry has increased its production rate without necessarily increasing food quality. High fructose corn syrup (HFCS), a product derived from cornstarch, is widely used in the manufacture of processed foods, and which excessive consumption is associated with an increase in the rate of obesity and metabolic disorders.

The metabolism of fructose, leads to the decrease of energy available in the organism. Simultaneously, metabolite production causes modifications in the cell signaling pathways inducing an increase in the quantity of lipids such as triglycerides and very low-density lipoprotein. High levels of these molecules promote conditions that lead to obesity and insulin resistance and that promote the development of type 2 diabetes mellitus, hyperuricemia and inflammation, which are the basis for the origin of cancer and neurological diseases. The present review focuses on the relationship between fructose and such maladies and on the possible action mechanisms.
\end{abstract}

Keywords: fructose, hyperuricemia, type 2 diabetes mellitus, lipogenesis, inflammation. 


\section{INTRODUCCIÓN}

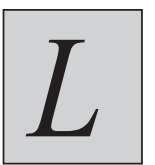

a fructosa es uno de los hidratos de carbono común de encontrar en muchos alimentos, tanto naturales como la miel, frutas y verduras, como en los procesados artificialmente, en los que se emplea en forma de Jarabe de Maíz Alto en Fructosa (JMAF) y cuando su consumo es en exceso y de manera crónica, el metabolismo de este monosacárido puede derivar en acciones que llevan a comprometer la salud. En esta revisión se describen los efectos de la fructosa en el desarrollo de padecimientos de alta incidencia en nuestro país como son: la hiperuricemia, el síndrome metabólico, la obesidad, la diabetes tipo 2, la neuro inflamación y el cáncer.

\section{MODELO EXPERIMENTAL}

La presente investigación es una revisión sistemática de 53 estudios científicos sobre la relación entre la alta ingesta de fructosa y las desregulaciones metabólicas que ésta ocasiona. Para evitar posibles sesgos, la estrategia de búsqueda en la selección de artículos se realizó de forma independiente por dos de los investigadores y se llegó a un acuerdo de los resultados vertidos con la ayuda de un tercer investigador.

\section{CONCEPTO E HISTORIA DEL JMAF}

El JMAF es un edulcorante líquido e incoloro procedente del maíz. Su obtención es a través de hidrólisis e isomerización del almidón contenido en el grano de maíz, por medio de un proceso enzimático (Soria, 2010). En 1966, en Japón se comenzó la elaboración de jarabes fructosados para la industria alimentaria por la compañía Sanmatsu Kogyo y, al año siguiente en Estados Unidos Clinton Corn Processing Company inicia la elaboración en América. Sin embargo, el verdadero cambio se da en 1972 cuando a los jarabes elaborados se les agrega una enzima catalizadora que los convierte en un glúcido ideal para la industria alimentaria por su fácil manejo, alto poder endulzante y precio menor al azúcar, convirtiéndose por lo mismo en el principal ingrediente utilizado por la industria en ese momento. Adicional a las características antes mencionadas el JMAF mostró una particularidad innovadora, ya que se etiquetó como no metabolizable o difícil de metabolizar. (García, Quintero \& López-Munguía, 2004).

\section{Proceso de obTención del JMAF}

El proceso comienza con el cultivo y la cosecha del maíz. Después se lleva a cabo la molienda y el cribado aislamiento del almidón que permite separarlo de las proteínas mediante la adición de agua y centrifugación para enseguida descomponerlo por medio de enzimas en glucosas, o sea, moléculas más pequeñas. El siguiente paso es la isomerización o adición de las enzimas amilasas: la alfa-amilasa que hidroliza al almidón en unidades de maltosa, la glucamilasa rompe las cadenas cortas derivadas de la acción de la alfa-amilasa para formar unidades de glucosa y por último la glucosa isomerasa que convierte a la glucosa en fructosa. Finalmente se purifica el jarabe con los métodos de filtración y evaporación dando como resultado un jarabe espeso y transparente con una concentración de fructosaglucosa en proporción de 42:58 o 55:45 (ver Figura 1) y listo para ser empleado como edulcorante y/o para mejorar la textura, el color y el sabor en los alimentos procesados.

\section{Alimentos PROCESAdos}

El ser humano ha empleado una serie de mecanismos para modificar los alimentos crudos con la finalidad de que sean aptos para su consumo, preparación o almacenamiento, a esta transformación se le llama procesamiento de alimentos. Los alimentos procesados han sido clave en la evolución, adaptación, bienestar y protección de la salud en las sociedades. A raíz de la industrialización de mediados del siglo XX, los alimentos también formaron parte del desarrollo tecnológico. Esto ha representado cambios en los patrones dietéticos de la sociedad, arrojando una balanza inclinada hacia un mayor consumo de alimentos transformados que se encuentran listos para ser ingeridos, y al mismo tiempo un menor consumo de alimentos frescos o tradicionales. Los alimentos procesados ganaron mayor aprobación entre la sociedad urbana y rural, hasta llegar a ser los de primera elección por la mayoría de las personas. Los principales demandantes y ofertantes, son las cadenas de comida rápida y los supermercados, (ver Tabla I) que venden alimentos con mayor cantidad de fructosa por porción respecto a los de origen natural (ver Tabla II). En 2016, se presentó una clasificación de alimentos por parte del epidemiólogo brasileño Carlos A. Monteiro y su grupo de colaboradores, tomando en cuenta su grado, orden y tipo de proceso que reciben durante su producción. Esta clasificación es conocida como NOVA(ver Tabla III). La clasificación NOVA se sustenta en los procesos físicos, químicos y biológicos que se llevan a cabo después de la extracción de los alimentos de la naturaleza y antes de que sean consumidos o empleados en la preparación de platillos o comida (Baquero, 2018).

\section{Metabolismo de la fRuctosa}

Aunque la glucosa y la fructosa comparten la misma fórmula química $\left(\mathrm{C}_{6} \mathrm{H}_{12} \mathrm{O}_{6}\right)$, la estructura química difiere en la sustitución de un grupo desde la posición del carbono 1 en la glucosa a la posición 2 en la fructosa, además, un átomo de hidrógeno de la glucosa está sustituido por un $\mathrm{CH}_{2} \mathrm{OH}$ en la fructosa. La diferencia es que la glucosa es una aldosa y la fructosa es una cetosa (es decir, el grupo funcional de la glucosa es un aldehído y de la fructuosa es un grupo ceto). Estas simples modificaciones estructurales confieren propiedades distintas desde la absorción hasta el metabolismo, con implicaciones en las funciones celulares de la fructosa (Theytaz et al., 2014).

El consumo en exceso de fructosa va más allá de las calorías que esta puede contener, sobre todo en la participación de las alteraciones bioquímicas que promueven el síndrome metabólico, condición de hallazgos fisiopatológicos que reúne a un grupo de factores que ponen en riesgo de contraer 


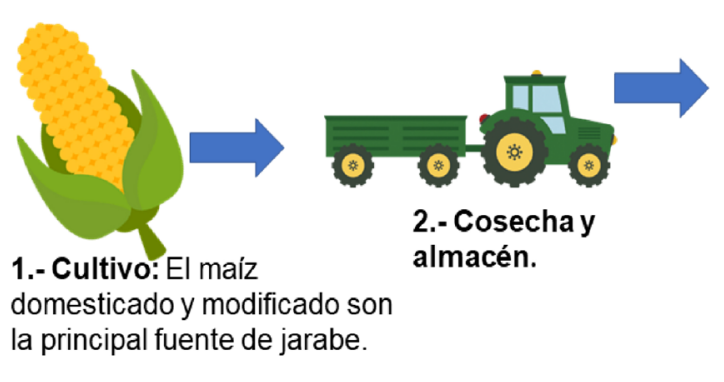

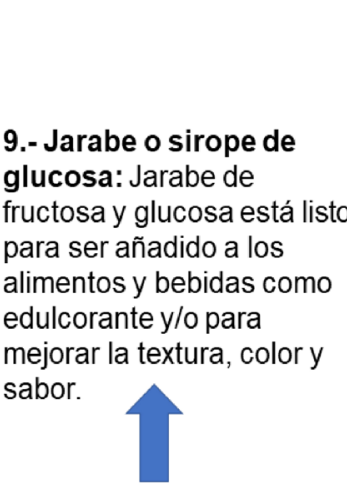

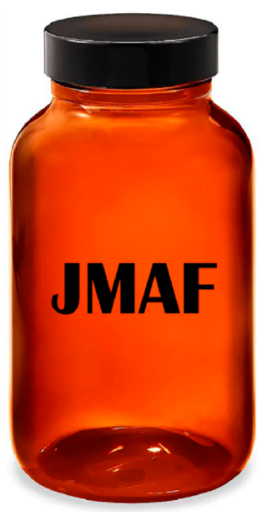

1010 3.- Molienda: É preparándose para la siguiente etapa

\section{8.-Purificación: El} jarabe se purifica mediante varios procesos adicionales de filtración y evaporación. Lo anterior da como resultado un jarabe espeso y transparente con una combinación de fructosaglucosa 42:58 o 55:45.
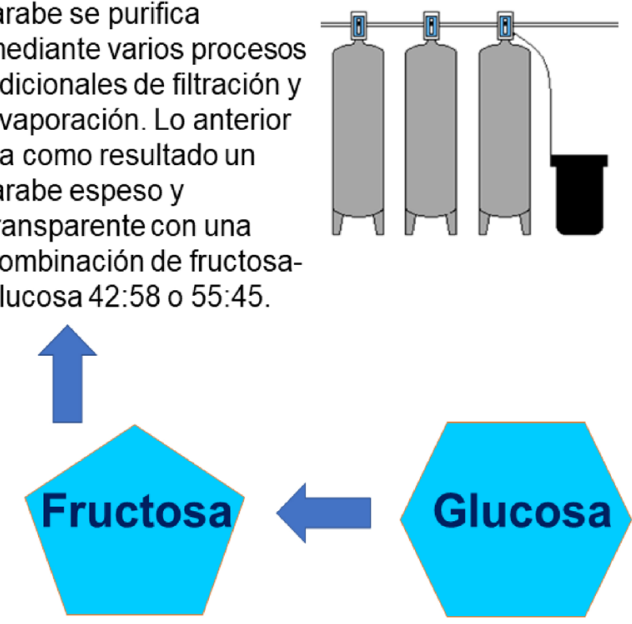

7.- Isomerización: La isomerización de la glucosa a fructosa se lleva a cabo utilizando 3 diferentes enzimas.

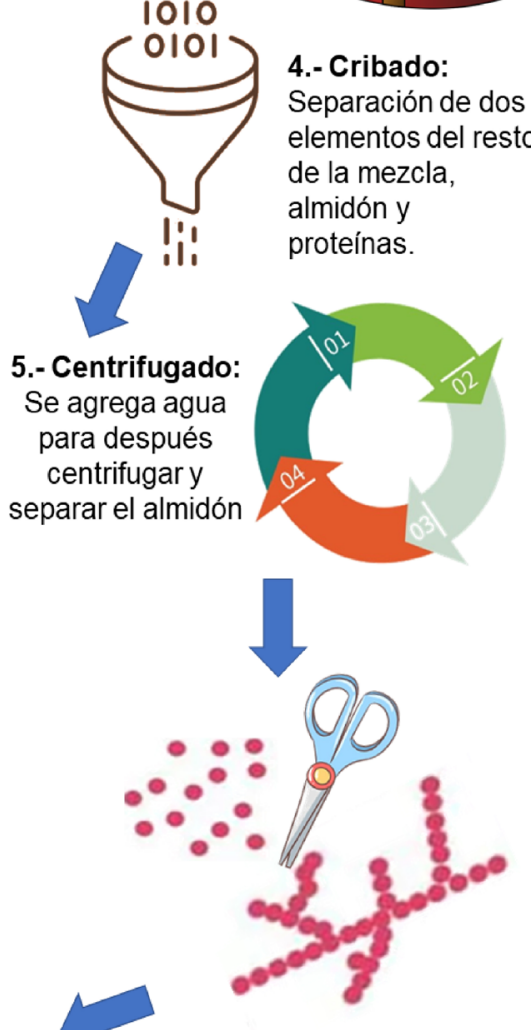

6.- Descomposición del almidón: El almidón es la unión de moléculas de glucosa. Esta unión es separada a través de enzimas. Así se obtiene el jarabe de glucosa.

Figura 1. Proceso de obtención del jarabe de maíz alto en fructosa. Proceso que describe los pasos para la obtención del jarabe de maíz alto en fructosa. Modificado de García, Quintero \& López-Munguía (2004).

enfermedades como la cardiaca, diabetes, obesidad y otros problemas de salud. El consumo de fructosa crea perfiles metabólicos distintos tanto en tejidos individuales como sistémicos (Mayes, 1993; Kovačević, Nestorov, Matić \& Elaković, 2014; Jiang et al., 2016). Una vez ingerida la fructosa, se transporta de forma independiente a la insulina mediante la membrana de los enterocitos intestinales a través de su transportador específico GLUT5 que se encuentra ubicado en la cara apical del enterocito, viajando así, por la circulación de la vena porta hacia el hígado. Una vez que la fructosa ingresa mediante el transportador GLUT2 en el hepatocito, esta se fosforila por la enzima fructocinasa, (FFK, por sus siglas en inglés) (Dornas, Lima, Pedrosa \& Silva, 2015), y posteriormente es cortada por la aldolasa B para formar gliceraldehído y dihidroxiacetona -fosfato, (DHA-P, dihydroxyacetone phosphate por sus siglas en inglés). Tanto el gliceraldehído como la dihidroxiacetona-fosfato son convertidos a la triosa gliceraldehído-3-fosfato (G-3-P, glyceraldehyde-3-phosphate 
Tabla I. Principales alimentos procesados que contienen JMAF.

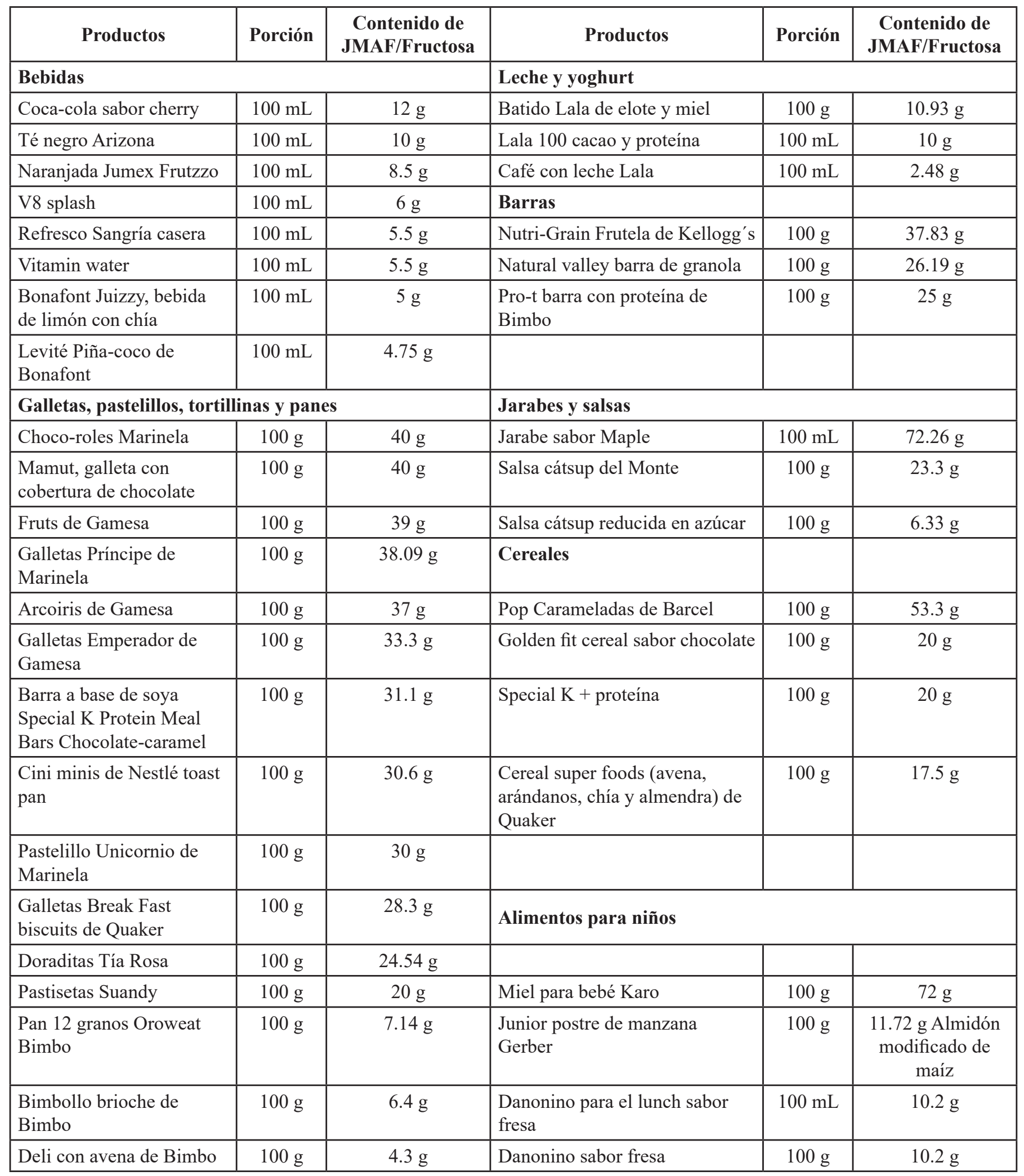

Cabada (2019). 
Tabla II. Principales alimentos de origen natural que contienen fructosa.

\begin{tabular}{|l|l|c|}
\hline \multicolumn{1}{|c|}{ Productos } & \multicolumn{1}{c|}{ Porción } & $\begin{array}{c}\text { Contenido de } \\
\text { Fructosa }\end{array}$ \\
\hline Miel & 1 cucharada & $9 \mathrm{~g}$ \\
\hline Pasas & $1 / 4$ de taza & $12 \mathrm{~g}$ \\
\hline Pera & mediana & $11 \mathrm{~g}$ \\
\hline Manzana & mediana & $11 \mathrm{~g}$ \\
\hline Arándanos & 1 taza & $7 \mathrm{~g}$ \\
\hline Plátano & mediano & $6 \mathrm{~g}$ \\
\hline Sandía & 1 taza & $5 \mathrm{~g}$ \\
\hline Uvas & 10 uvas & $4 \mathrm{~g}$ \\
\hline Fresas & 1 taza & $4 \mathrm{~g}$ \\
\hline Pepino & mediano & $3 \mathrm{~g}$ \\
\hline Tomate & mediano & $2 \mathrm{~g}$ \\
\hline
\end{tabular}

Dahl, Foster \& Owen (2020). por sus siglas en inglés) (Bray, 2013). Debido a que la fructólisis no es regulada como en el caso de la glucólisis por las enzimas hexocinasa y fosfofructocinasa, grandes cantidades de fructosa son convertidas a lactato, glucosa y ácidos grasos en el hígado, independientemente de la cantidad de energía disponible (Tappy \& Lê, 2010), Figura 2.

Debido a que la fructosa no estimula la liberación directamente de la insulina por las células beta-pancreáticas como lo hace la glucosa, la producción endógena tiene un incremento que conlleva a la regulación por la fructosa que actúa como un potente activador de la glucosa 6 -fosfatasa, enzima que interviene en la inhibición de la gluconeogénesis. Considerando estos mecanismos de acción de la fructosa, una dieta rica en este hidrato de carbono puede conducir a la resistencia a la insulina en el hígado (Koo et al., 2008; Zavaroni, Sander, Scott \& Reaven, 1980). Aunado a esto, un catabolismo desregulado de la fructosa puede influir en el metabolismo hepático y sistémico de la glucosa y de los ácidos grasos, provocando enfermedades como la diabetes e hipertensión.

Tabla III. Clasificación de alimentos NOVA.

\begin{tabular}{|c|c|}
\hline $\begin{array}{l}\text { Alimentos no procesados o mínimamente } \\
\text { procesados (NP o MP) }\end{array}$ & $\begin{array}{l}\text { Agua corriente (de grifo): filtrada, de manantial o mineral. Carnes: aves } \\
\text { de corral. Pescados o mariscos: frescos, secos, refrigerados o congelados. } \\
\text { Especias. Tés, café e infusiones de hierbas. Frutos secos y semillas sin sal } \\
\text { ni azúcares añadidos. Harinas: pastas alimenticias crudas hechas de harina y } \\
\text { agua. Jugos de fruta: recién preparados o pasteurizados sin azúcares añadidos, } \\
\text { edulcorantes ni saborizantes. Leche: en polvo, fresca, entera, parcial o } \\
\text { totalmente descremada, pasteurizada y leche fermentada. Yoghurt: natural sin } \\
\text { azúcares añadidos ni edulcorantes artificiales. Raíces, tubérculos y hongos. } \\
\text { Verduras, frutas, cereales. Legumbres: frescas, refrigeradas, congeladas, } \\
\text { envasadas al vacío o desecadas. }\end{array}$ \\
\hline $\begin{array}{l}\text { Alimentos con Ingredientes culinarios procesados } \\
\text { (ICP) }\end{array}$ & $\begin{array}{l}\text { Aceites vegetales, mantequilla y manteca, almidones, azúcar, melaza, miel y } \\
\text { jarabes, sal de roca, marina o yodada, vinagre de vino y bebidas alcohólicas. }\end{array}$ \\
\hline Alimentos procesados (P) & $\begin{array}{l}\text { Carnes y pescados: procesados, salados o curados (jamón) o en conserva. } \\
\text { Cerveza, sidra o vino. Frutas en almíbar, secas y semillas saladas o azucaradas. } \\
\text { Panes elaborados con harinas, agua, sal y fermentos. Quesos hechos con leche, } \\
\text { sal y fermentos. Verduras y legumbres enlatadas o embotelladas. }\end{array}$ \\
\hline Alimentos ultra - procesados (UP) & $\begin{array}{l}\text { Bebidas: gaseosas azucaradas, 'energéticas', azucaradas a base de leche o } \\
\text { cacao y néctares de frutas; cerveza/vino sin alcohol, whisky, ginebra, ron y } \\
\text { vodka. Fideos/sopas: enlatadas, embotelladas, deshidratadas o empaquetadas, } \\
\text { fórmulas infantiles y leches de continuación, helados, chocolate, dulces y } \\
\text { caramelos, mermeladas, jaleas, margarinas y salsas. Panes y bollos empacados } \\
\text { producidos a nivel industrial. Platos de: carne, pescado, vegetales, queso, } \\
\text { pasta o pizza ya preparados. Snacks, galletas, cereales de desayuno, barritas } \\
\text { de 'cereales' y 'energéticas', pasteles y tartas, sustitutivos de comidas y } \\
\text { alimentos fortificados. Yoghurt: natural o de frutas con azúcares añadidos o } \\
\text { edulcorantes artificiales. }\end{array}$ \\
\hline
\end{tabular}

Baquero (2018). 


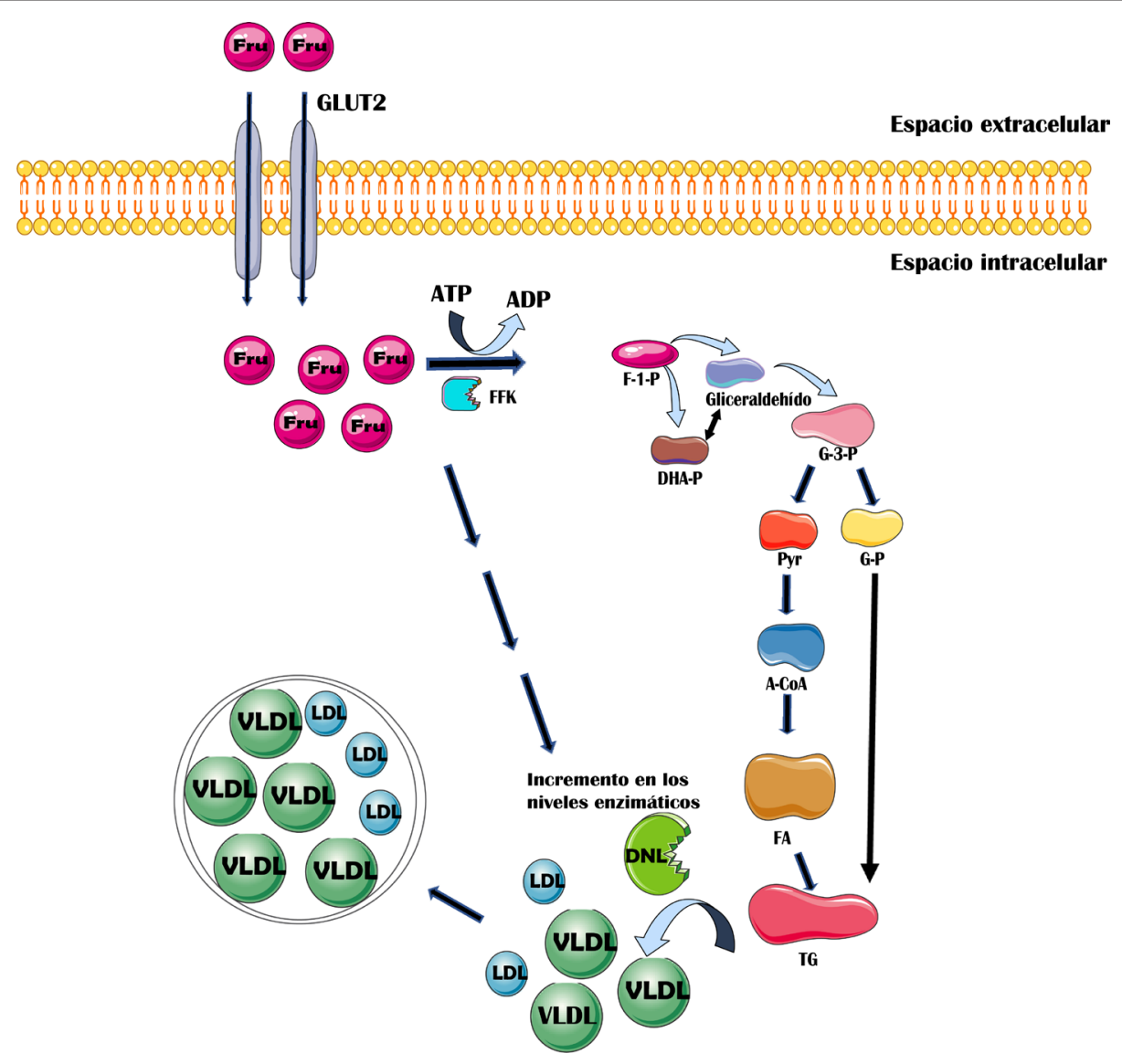

Figura 2. Metabolismo de la fructosa en el hígado. El metabolismo de la fructosa en concentraciones normales para participar en el ciclo de los ácidos tricarboxílicos por demanda del organismo, o bien, cuando las concentraciones se encuentran en exceso puede ser utilizada en la vía de la lipogénesis de novo. Fructosa (Fru), Fosfofructocinasa (FFK), Fructosa-1-fosfato (F-1-P), Dihidroxiacetona-fosfato (DHA-P), Gliceraldehído-3-fostafo (G-3-P), Piruvato (Pyr), Glicerol-fosfato (G-P), Acetil-Coenzima A (A-CoA), Ácidos grasos (FA), Triglicéridos (TG), De Novo Lipogénesis (DNL), Lipoproteína de Baja Densidad (LDL), Lipoproteína de Muy Baja Densidad (VLDL). Modificado de Carvallo et al. (2019).

\section{LA FRUCTOSA EN LA LIPOGÉNESIS}

Se sabe que las dietas altas en fructosa aumentan los lípidos hepáticos, por ejemplo, los niveles séricos de los triglicéridos, lipoproteína de baja densidad (LDL, low density lipoprotein, por sus siglas en inglés) y lipoproteína de muy baja densidad (VLDL, very low density lipoprotein, por sus siglas en inglés) Figura 3 a, con incremento de la adiposidad visceral en humanos y en roedores (Stanhope et al., 2009; Pektaş, Sadi \& Akar, 2015). Por su mecanismo de acción, la fructosa incrementa las enzimas críticas involucradas en la lipogénesis de novo (DNL, de novo lipogénesis, por sus siglas en inglés). Este monosacárido regula de manera positiva la producción de las enzimas ácido graso sintasa y glicerol 3 -fosfato aciltransferasa en los adipocitos, catalizando el primer paso en la síntesis de glicerolípidos, e incrementando el número o tamaño de las vesículas lipídicas que conducen a la obesidad (Robubi, Huber \& Krugluger, 2014). Aunado a este proceso de generación y acumulación de ácidos grasos, la xilulosa 5-fosfato (Xu-5-P, xylulose 5-phosphate, por sus siglas en inglés) metabolito de la vía de las hexosas monofosfato activa a la proteína fosfatasa 2A para regular los efectos agudos de la alimentación de hidratos de carbono en la vía glucolítica, así como el control coordinado a largo plazo de las enzimas requeridas para la síntesis de ácidos grasos y triglicéridos. La alimentación con hidratos de carbono incrementa los niveles de glucosa en el hígado y fructosa 6-fosfato (Fru-6-P, fructose 6-phosphate por sus siglas en inglés). La elevación de la Xu-5-P es la señal de coordinación para activar de forma aguda a la fosfofructocinasa en la glucólisis y promover la acción de la proteína de unión al elemento sensible a hidratos de carbono del factor de transcripción (ChREBP) para aumentar la transcripción de los genes en las enzimas de la lipogénesis, de las hexosas monofosfato y la glucólisis, todos necesarios para la síntesis de novo en las grasas (Veech, 2003). 


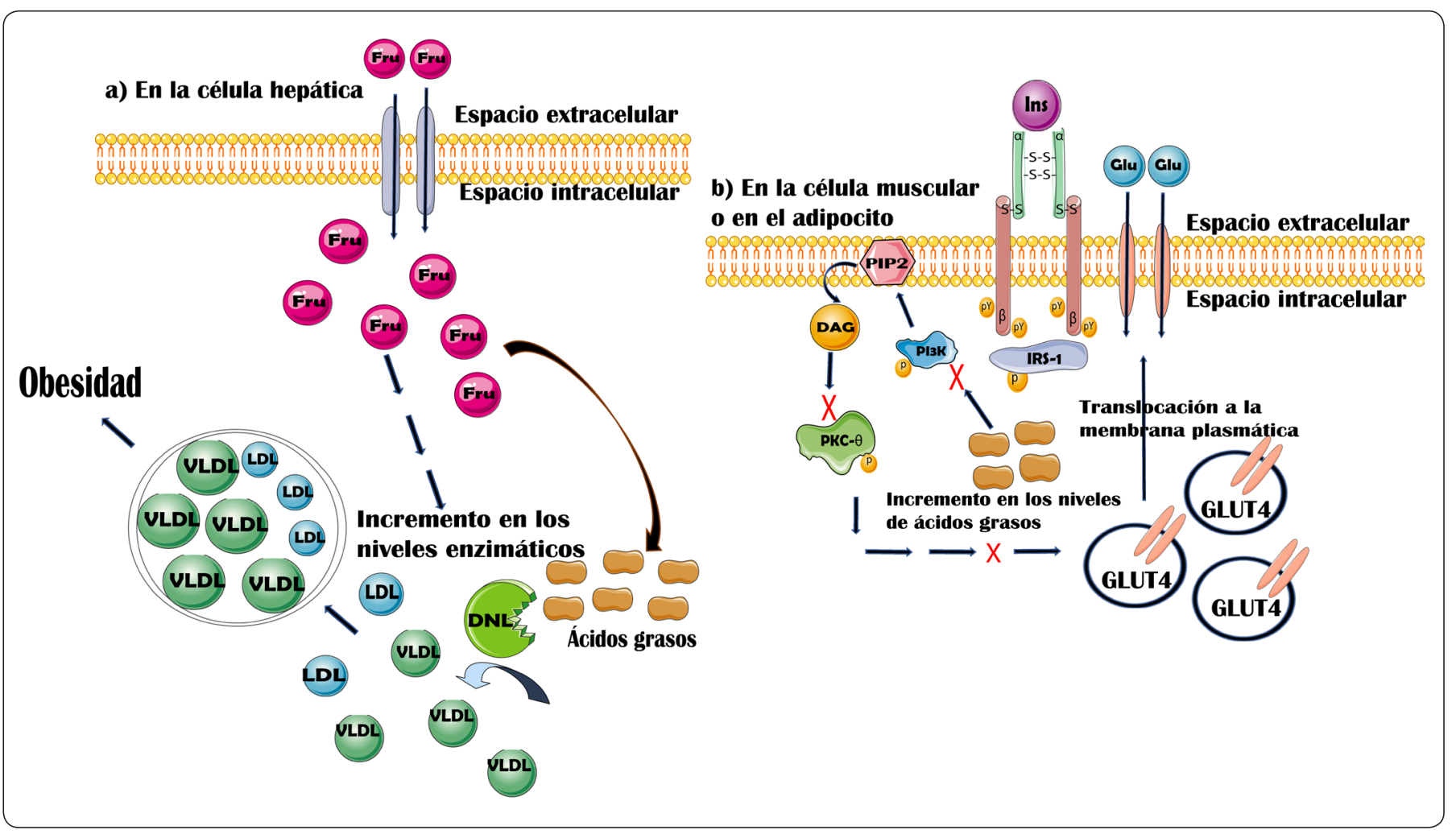

Figura 3. Lipogénesis y resistencia a la insulina ocasionada por el consumo de fructosa. En los hepatocitos el alto nivel de fructosa en el espacio intracelular conlleva a la generación de ácidos grasos y al incremento de los niveles enzimáticos de DNL y a su a vez a la presencia intracelular de LDL y VLDL implicando obesidad (a). En las células musculares o en los adipocitos el incremento del DAG evita que se fosforile y active la isoforma PCKO previniendo la translocación del GLUT4 a la membrana plasmática y contribuyendo a la inhibición de la señalización el alto nivel de ácidos grasos interrumpe la activación de la PI3K provocando resistencia a la insulina (b). Insulina (Ins), Sustrato del receptor de insulina-1 (IRS-1), Fosfatidil Inositol 3- Cinasa (PI3K), de novo Lipogénesis (DNL), DiAcil Glicerol (DAG), Proteína Cinasa C teta (PCKO), Transportador 4 de glucosa (GLUT4), Glucosa (Glu), Fructosa (Fru), Lipoproteína de Baja Densidad (LDL), Lipoproteína de Muy Baja Densidad (VLDL). Modificado de OlivaresReyes \& Arellano-Plancarte (2008).

\section{LA FRUCTOSA EN EL MECANISMO DE RESISTENCIA A LA INSULINA}

La afección vinculada con la prediabetes y la diabetes mellitus tipo 2 conocida como resistencia a la insulina, se debe a que el organismo es incapaz de responder a ella, independientemente de la cantidad de insulina que se produce. Las primeras alteraciones básicas inducidas por la fructosa son el aumento del estrés oxidante, la glicosilación de proteínas, la inflamación y la resistencia a la insulina (Gatineau, Polakof, Dardevet \& Mosoni, 2017). A nivel subcelular, la disminución de la beta oxidación mitocondrial de los ácidos grasos y a su vez, la acumulación de diacilglicerol (DAG, diacylglycerol por sus siglas en inglés) inhibe la vía de señalización de la insulina a nivel de la proteína cinasa C (PKC, protein kinase C por sus siglas en inglés) (Samuel et al., 2004). En particular, una de las isoformas de la PKC, la PKC teta ( $\mathrm{PKC} \theta$, protein kinase teta por sus siglas en inglés), ocasiona un deterioro en la señalización del receptor de insulina (IRS-1, insulin receptor substrate-1 por sus siglas en inglés) manifestándose en diabetes tipo 2, interrumpiendo la señalización correcta de la insulina y con ello las acciones mediadas de esta hormona (Krssak et al., 2004; Szendroedi et al., 2014).

La alta concentración de fructosa promueve el desarrollo del síndrome metabólico mediante el incremento del tejido adiposo, lo que conduce a un aumento en los niveles de ácidos grasos libres en la circulación portal (Rutledge \& Adeli, 2008), ya que en la señalización río abajo del receptor de insulina, la actividad de la fosfatidil inositol-3-cinasa (PI3K, phosphatidylinositol-3kinasa por sus siglas en inglés) asociada al IRS-1, no muestra respuesta cuando existe un pretratamiento con ácidos grasos libres en las células musculares (Dresner et al., 1999; Pektaş et al., 2015) Figura 3 b. Por consiguiente, el aumento resultante en la captación hepática de estos ácidos grasos libres conduce a la fácil disponibilidad de los lípidos en el hígado promoviendo la resistencia a la insulina (Bergman et al., 2007). 


\section{LA FRUCTOSA EN LA HIPERURICEMIA}

Existen una gran variedad de factores en la dieta que pueden ser promotores de la hiperuricemia mediante la elevación de las purinas, como ocurre en la degradación de ciertas proteínas ingeridas con los alimentos. La fructosa como ingrediente dietético popular en alto consumo puede contribuir a la hiperuricemia mediante la estimulación del catabolismo de los nucleótidos de adenina (Cicero et al., 2019). Asimismo, la evidencia sugiere que los azúcares agregados, principalmente la fructosa tiene un impacto desfavorable sobre el metabolismo provocando gota, hiperuricemia, obesidad, hipertensión, hígado graso no alcohólico y diabetes tipo 2 (Carvallo et al., 2019). El incremento de los uratos está mediado por la actividad de la enzima cetohexocinasa (KHK, ketohexoquinase, por sus siglas en inglés) que, a diferencia de otras cinasas, tiene la capacidad de inducir el agotamiento transitorio del trifosfato de adenosina (ATP, adenosine triphosphate, por sus siglas en inglés) como consecuencia de su rápida fosforilación de fructosa a fructosa1-fosfato. Dado que el metabolismo de la fructosa ocurre en el hígado, este agotamiento de ATP afecta a otros procesos metabólicos en el organismo. El agotamiento de ATP en la célula conduce a la depleción del fosfato y como consecuencia ocurre un incremento en los niveles de monofosfato de adenosina (AMP, adenosine monophosphate, por sus siglas en inglés). Este fenómeno conlleva a la actividad catabólica de la enzima AMP desaminasa que resulta en la última degradación del AMP para producir urato, por lo tanto, el urato es el producto final del nucleótido de purina. El catabolismo y la hiperuricemia se caracterizan por un exceso en la producción y deposición de cristales de urato que conducen al padecimiento conocido como gota (Cox et al., 2012).

\section{LA FRUCTOSA EN LA INFLAMACIÓN}

El tejido adiposo secreta adipocinas y citocinas (factores responsables de la inflamación) que pueden afectar al metabolismo y al sistema inmunológico. Debido a que el consumo de fructosa promueve la obesidad, puede también de manera directa o indirecta promover la secreción desregulada de estos factores. En el hígado y el sistema digestivo, la fructosa promueve la inflamación y reduce la permeabilidad intestinal (Bergheim et al., 2008). Es en el hígado donde la acumulación de ácidos grasos libres induce a estrés oxidante e incrementa la actividad transcripcional del Factor Nuclear kappa B, (NFкB, nuclear factor k B, por sus siglas en inglés), que es clave en los procesos inflamatorios (Roglans et al., 2007). En condiciones de obesidad se encuentra elevado en el plasma el factor de necrosis tumoral, (TNF, tumor necrosis factor, por sus siglas en inglés) como se observó en los ratones al alimentarlos con fructosa (Spruss et al., 2009) y en hámsters (Tsai et al., 2009). Adicionalmente, existe una pérdida en la función endotelial provocada por la fructosa y esta pérdida se relaciona con una infiltración de macrófagos y células $\mathrm{T}$ en el tejido adiposo perivascular (Jia, Aroor, Whaley-Connell \& Sowers, 2014).

\section{LA FRUCTOSA EN EL ESTRÉS CELULAR Y EL CÁNCER}

Debido a que el consumo de fructosa produce un incremento del tejido adiposo, éste último puede coordinar señales mediante la secreción de adipocinas, donde muchas de ellas están involucradas con el metabolismo y reprogramación de células cancerosas. Se sabe que la citocina TNF, la quimiocina CCL-2 (CCL-2, chemokine C-C motif ligand 2, por sus siglas en inglés) y el factor FGF (FGF, fibroblast growth factor, por sus siglas en inglés) están implicados directamente en la progresión del cáncer de mama (Strober \& Brady, 2019; Johnson et al., 2009; Kammoun et al., 2009). Durante el estrés oxidante, el eje hipotalámico-pituitario-adrenal (HPA, hypothalamicpituitary-adrenal, por sus siglas en inglés) estimula la secreción del cortisol que se une al receptor de los glucocorticoides estimulando las vías de señalización anti-apoptóticas (Lêet al., 2006) promoviendo de esta forma las condiciones para el inicio o desarrollo de un tumor. Existen estudios que demuestran que en un modelo experimental de ratas alimentadas con una dieta alta en fructosa desarrollaron daño oxidante hepático como resultado de la carga del metabolismo de la fructosa hasta conducir al cáncer y si las personas presentan bajos niveles del inhibidor de la apoptosis en los macrófagos, serán más susceptibles cuando la ingesta de fructosa es crónica (Ozawa, Maehara, Kai, Arai \& Miyazaki, 2016).

Otro tipo de cáncer que se encuentra asociado al consumo de fructosa, es el cáncer de páncreas. El consumo de cantidades de fructosa $>25 \mathrm{~g}$ /día durante un periodo de seguimiento de al menos 8 años puede incrementar el riesgo de padecer cáncer de páncreas (Nöthlings, Murphy, Wilkens, Henderson \& Kolonel, 2007; Aune et al., 2012; Genkinger et al., 2011). El intestino también está en el radar del cáncer como consecuencia del consumo de fructosa, ya que se ha demostrado que en ratones la cantidad de fructosa está incrementada en la luz intestinal y en los tumores de estas células intestinales (Goncalves et al., 2019).

El mecanismo específico que explica la asociación entre un elevado consumo de fructosa y el cáncer se desconoce aún, sin embargo, existe evidencia de que la fructosa contribuye en la síntesis de ácidos nucleicos a través de la vía de las hexosas monofosfato, catalizada por la enzima transcetolasa (TKT, transketolase, por sus siglas en inglés) (Liu et al., 2010), siendo la síntesis de RNA y DNA necesarios para la proliferación celular y en especial de las células cancerosas, además de la activación de la transcetolasa que estimula el crecimiento del tumor (Comín-Anduix et al., 2001). Dentro de los tumores, la fructosa se convierte en fructosa-1-fosfato activando la vía de la glucólisis para producir gliceraldehido 3-fosfato y conducir a su vez a la síntesis de ácidos grasos que apoyan al crecimiento tumoral (Goncalves et al., 2019). Existe evidencia de estudios que señalan que la fructosa se puede utilizar como fuente de carbono en algunos tipos de cáncer para mantener la supervivencia y proliferación celular. El cáncer en el cerebro muestra también relación con la fructosa, ya que la enzima 
KHK se encuentra expresada significativamente más alta en los tejidos del glioma respecto al tejido cerebral no tumoral y esta expresión de la enzima se correlaciona significativamente con tumores malignos y una pobre sobrevivencia de los pacientes con glioma (Gao, Li, Li, Xu \& Su, 2018).

\section{LA FRUCTOSA EN LAS ENFERMEDADES NEUROLÓGICAS}

Los efectos de la fructosa en el cerebro pueden ser de mayor alcance por su capacidad de generar resistencia a la insulina. La resistencia a esta hormona en el cuerpo puede interrumpir la señalización del receptor de insulina en el cerebro y con ello la aparición de desórdenes neurodegenerativos (Shulman, 2000). El RNA mensajero del transportador de fructosa, el transportador GLUT5, se encuentra mayormente expresado en el cerebro, específicamente en el hipocampo, (Shu, Isenberg, Cormier, Benz \& Zorumski, 2006), lo que implica que la captación neuronal de la fructosa se puede aumentar en ratas alimentadas con este endulzante (Lindqvist, Baelemans \& Erlanson-Albertsson, 2008). A nivel neuronal, una dieta alta en fructosa en conjunto con un incremento de lípidos en la sangre, ocasiona un cambio en la expresión de los genes en el hipocampo como el gen $I L 18$, que codifica para la citocina proinflamatoria IL-18, y de Lcn2, gen involucrado en la activación de los polimorfonucleares, que lleva a este mecanismo inflamatorio a impactar en el comportamiento de una conducta deficiente, determinada por la capacidad de exploración y sociabilización (De Sousa et al., 2017). Adicionalmente, el consumo de fructosa también afecta la expresión de las citocinas proinflamatorias como la IL-6 e IL-1beta en el hipocampo, con un impacto en el aprendizaje espacial y en la ansiedad en la etapa adolescente (Hsu et al., 2014).

Cada vez se genera mayor evidencia que sugiere que los diagnósticos psiquiátricos, especialmente el trastorno bipolar es significativamente más común en pacientes que presentan diabetes tipo 2 (Wandell, Ljunggren, Wahlström \& Carlsson, 2014; Charles, Lambert \& Kerner, 2016). El consumo de alta fructosa en ratas adolescentes conduce a un fenotipo conductual de tipo bipolar, con hiperexcitabilidad neuronal que se sabe que es una de las primeras manifestaciones endofenotípicas del trastorno bipolar (Alten et al., 2018).

\section{Conclusiones}

Actualmente, es posible estar expuestos a altas ingestas de fructosa en forma de JMAF con los subsecuentes daños a la salud, ya que el metabolismo de la fructosa ocurre mayormente en el hígado y se necesita en un inicio el aporte de energía, de esta manera se genera un agotamiento energético que lleva de manera directa a la producción de hiperuricemia y a la afectación de otros procesos metabólicos. La acumulación de los ácidos grasos que se originan por el metabolismo mencionado tiene tres destinos: el primero es el que interrumpe la señalización del receptor de insulina, evitando la translocación del transportador GLUT4 para la entrada de la glucosa, desembocando en la resistencia a la insulina; el segundo es el que conduce directamente al incremento en el número o tamaño de las vesículas lipídicas, ocasionando obesidad y el tercero es el que incrementa la actividad inflamatoria del factor de transcripción NFкB. El proceso inflamatorio, es la antesala para que se incremente la probabilidad de la implantación y desarrollo del cáncer y las enfermedades neurológicas. Las evidencias vertidas en este artículo apuntan a que es posible encontrar la respuesta en la cantidad de fructosa que se ingiere de manera habitual en los alimentos, crea dependencia, y al consumirla en exceso compromete la salud.

\section{Agradecimientos}

El agradecimiento de los autores se hace extensivo a María del Refugio Espíndola Ronquillo, Roberto Quiroga Osornio y Guillermo Isaac Gómez Fuentes por el apoyo en la búsqueda de las referencias bibliográficas.

\section{DECLARACIÓN DE CONFLICTO DE INTERESES}

Los autores declaran que no tienen conflicto de intereses.

\section{REFERENCIAS}

Alten, B., Yesiltepe, M., Bayraktar, E., Tas, S. T., Gocmen, A. Y., Kursungoz, C., Martinez, A. \& Sara, Y. (2018). High fructose corn syrup consumption in adolescent rats causes bipolar-like behavioural phenotype with hyperexcitability in hippocampal CA3-CA1 synapses. British Journal of Pharmacology, 175(24), 4450-4463. DOI:10.1111/ bph. 14500.

Aune, D., Chan, D. S. M., Vieira, A. R., Navarro Rosenblatt, D. A., Vieira, R., Greenwood, D. C., Cade, J. E., Burley, V. J. \& Norat, T. (2012). Dietary fructose, carbohydrates, glycemic indices and pancreatic cancer risk: a systematic review and meta-analysis of cohort studies. Annals of Oncololgy, 23(10), 2536-2546. DOI:10.1093/annonc/mds076.

Baquero, A. (2018).Alimentos ultraprocesados y su impacto en la dieta actual. Madrid. Universidad complutense de Madrid.

Bergheim, I., Weber, S., Vos, M., Krämer, S., Volynets, V., Kaserouni, S., McClain, C. J. \& Bischoff, S. C. (2008). Antibiotics protect against fructose-induced hepatic lipid accumulation in mice: Role of endotoxin. Journal of Hepatology, 48(6), 983-992. DOI: 10.1016/j. jhep.2008.01.035.

Bergman, R. N., Kim, S. P., Hsu, I. R., Catalano, K. J., Chiu, J. D., Kabir, M., Richey, J. M. \& Ader, M. (2007). Abdominal obesity: role in the pathophysiology of metabolic disease and cardiovascular risk. The American Journal of Medicine, 120(2), S3-S8. DOI: 10.1016/j.amjmed.2006.11.012.

Bray, G. A. (2013). Energy and fructose from beverages sweetened with sugar or high-fructose corn syrup pose a health risk for some people. Advances in Nutrition, 4(2), 220-225. DOI:10.3945/an.112.002816.

Cabada, X. (2019, noviembre). Jarabe de maíz de alta fructosa, el gran impostor del azúcar. Revista del consumidor, (513), 
38-43. Recuperado de: https:/issuu.com/profeco/docs/ revista_del_consumidor_noviembre_20_78e7e72f07c02b.

Carvallo, P., Carvallo, E., Barbosa-da-Silva, S., Mandarim-deLacerda, C.A., Hernández,A. \& del-Sol, M. (2019). Efectos metabólicos del consumo excesivo de fructosa añadida. International Journal of Morphology, 37(3), 10581066. DOI:10.4067/s0717-95022019000301058.

Cicero, A. F. G., Fogacci, F., Desideri, G., Grandi, E., Rizzoli, E., D’Addato, S. \& Borghi, C. (2019). Arterial stiffness, sugar-sweetened beverages and fruits intake in a rural population sample: Data from the Brisighella heart study. Nutrients, 11(11), 2674. DOI:10.3390/nu11112674

Charles, E. F., Lambert, C. G. \& Kerner, B. (2016). Bipolar disorder and diabetes mellitus: evidence for diseasemodifying effects and treatment implications. International Journal of Bipolar Disorders, 4(1), 13. DOI:10.1186/ s40345-016-0054-4.

Comín-Anduix, B., Boren, J., Martinez, S., Moro, C., Centelles, J. J., Trebukhina, R., Petushok, N., Lee, W. P., Boros, L. G. \& Cascante, M. (2001). The effect of thiamine supplementation on tumour proliferation. European Journal ofBiochemistry, 268(15), 4177-4182. DOI:10.1046/j.14321327.2001.02329.x.

Cox, C. L., Stanhope, K. L., Schwarz, J., Graham, J. L., Hatcher, B., Griffen, S. C., Bremer, A. A., Berglund, L., McGahan, J. P., Keim, N. L. \& Havel, P. J. (2012). Consumption of fructose- but not glucose-sweetened beverages for 10 weeks increases circulating concentrations of uric acid, retinol binding protein-4, and gamma-glutamyl transferase activity in overweight/obese humans. Nutrition \& Metabolism, 9(1), 68. DOI:10.1186/1743-7075-9-68

Dahl, W. J., Foster, L. \& Owen, R. J. (2020). Los hechos acerca de la fructosa. IFAS extensión, University of Florida.

De Sousa Rodrigues, M. E., Bekhbat, M., Houser, M. C., Chang, J., Walker, D. I., Jones, D. P., Oller do Nascimento, C. M. P., Barnum, C. J. \& Tansey, M. G. (2017). Chronic psychological stress and high-fat high-fructose diet disrupt metabolic and inflammatory gene networks in the brain, liver, and gut and promote behavioral deficits in mice. Brain Behavior Immunity, 59(1), 158-172. DOI: 10.1016/j. bbi.2016.08.021.

Dornas, W. C., de Lima, W. G., Pedrosa, M. L. \& Silva, M, E. (2015). Health implications of high-fructose intake and current research. Advances in Nutrition, 6(6), 729-737. DOI: 10.3945/an.114.008144.

Dresner, A., Laurent, D., Marcucci, M., Griffin, M. E., Dufour, S., Cline, G. W., Slezak, L. A., Andersen, D. K., Hundal, R. S., Rothman, D. L., Petersen, K. F. \& Shulman, G. I. (1999). Effects of free fatty acids on glucose transport and IRS-1-associated phosphatidylinositol 3-kinase activity. Journal of Clinical Investigation, 103(2), 253259. DOI:10.1172/jci5001.

Gao, W., Li, N., Li, Z., Xu, J. \& Su, C. (2018). Ketohexokinase is involved in fructose utilization and promotes tumor progression in glioma. Biochemical and Biophysical Research Communications, 503(3), 1298-1306. DOI: 10.1016/j.bbrc.2018.07.040.

García, G. M., Quintero, R. R. \& López-Munguía, C. A. (2004) Biotecnología alimentaria $\left(1^{\mathrm{a}}\right.$ ed.). [EPUB], Ciudad de México. México.

Gatineau, E., Polakof, S., Dardevet, D. \& Mosoni, L. (2017). Similarities and interactions between the ageing process and high chronic intake of added sugars. Nutrition Research Reviews, 30(02), 191-207. DOI:10.1017/ s0954422417000051.

Genkinger, J. M., Li, R., Spiegelman, D., Anderson, K. E., Albanes, D., Bergkvist, L., Bernstein, L., Black, A., Van den Brandt, P. A., English, D. R., Freudenheim, J. L., Fuchs, C. S., Giles, G. G., Giovannucci, E., Goldbohm, R. A., Horn-Ross, P. L., Jacobs, E. J., Koushik, A., Männistö, S., Marshall, J. R., Miller, A. B., Patel, A. V., Robien, K., Rohan, T. E., Schairer, C., Stolzenberg-Solomon, R., Wolk, A., Ziegler, R. G. \& Smith-Warner, S. A. (2011). Coffee, tea, and sugar-sweetened carbonated soft drink intake and pancreatic cancer risk: A pooled analysis of 14 cohort studies. Cancer Epidemiology Biomarkers \& Prevention, 21(2), 305-318. DOI: 10.1158/1055-9965.epi-11-0945-t.

Goncalves M. D., Lu, C., Tutnauer, J., Hartman, T. E., Hwang, S. K., Murphy, C. J., Pauli, C., Morris, R., Taylor, S., Bosch, K., Yang, S., Wang, Y., Van Riper, J., Lekaye, H., C., Roper, J., Kim, Y., Chen, Q., Gross, S. S., Rhee, K., Y., Cantley, L. C. \& Yun, J. 2019. High-fructose corn syrup enhances intestinal tumor growth in mice. Science, 363(6433), 1345 1349. DOI:10.1126/science.aat8515.

Hsu, T. M., Konanur, V. R., Taing, L., Usui, R., Kayser, B. D., Goran, M. I. \& Kanoski, S. E. (2014). Effects of sucrose and high fructose corn syrup consumption on spatial memory function and hippocampal neuroinflammation in adolescent rats. Hippocampus, 25(2), 227-239. DOI:10.1002/ hipo. 22368 .

Jia, G., Aroor, A. R., Whaley-Connell, A. T. \& Sowers, J. R. (2014). Fructose and uric acid: ¿Is there a role in endothelial function? Current Hypertension Reports, 16(6), 434. DOI:10.1007/s11906-014-0434-z.

Jiang, Y., Pan, Y., Rhea, P. R., Tan, L., Gagea, M., Cohen, L., Fischer, S. M. \& Yang, P. (2016). A sucrose-enriched diet promotes tumorigenesis in mammary gland in part through the 12-lipoxygenase pathway. Cancer Research, 76(1), 24-29. DOI: 10.1158/0008-5472.CAN-14-3432.

Johnson, R. K., Appel, L. J., Brands, M., Howard, B. V., Lefevre, M., Lusting, R. H., Sacks, F., Steffen, L. M. \& Wylie-Rosett, J. (2009). Dietary sugars intake and cardiovascular health: A scientific statement from the American Heart Association. Circulation, 120(11), 10111020. DOI:10.1161/circulationaha.109.192627.

Kammoun, H. L., Chabanon, H., Hainault, I., Luquet, S., Magnan, C., Koike, T., Ferré, P. \& Foufelle, F. (2009). GRP78 expression inhibits insulin and ER stress-induced SREBP-1c 
activation and reduces hepatic steatosis in mice. Journal of Clinical Investigation, 119(5), 1201-1215. DOI:10.1172/ jci37007.

Koo, H. Y., Wallig, M. A., Chung, B. H., Nara, T. Y., Cho, B. H. S.\& Nakamura, M. T. (2008). Dietary fructose induces a wide range of genes with distinct shift in carbohydrate and lipid metabolism in fed and fasted rat liver. Biochimica et Biophysica Acta, 1782(5), 341-348. DOI: 10.1016/j. bbadis.2008.02.007.

Kovačević, S., Nestorov, J., Matić, G. \& Elaković, I. (2014) Dietary fructose-related adiposity and glucocorticoid receptor function in visceral adipose tissue of female rats. European Journal of Nutrition, 53(6),1409-1420. DOI: 10.1007/s00394-013-0644-1.

Krssak, M., Brehm,A., Bernroider, E.,Anderwald, C., Nowotny, P., Man, C. D., Cobeli, C. Cline, G. W., Shulman, G. I., Waldhäusl, W. \& Roden, M. (2004). Alterations in postprandial hepatic glycogen metabolism in type 2 diabetes. Diabetes, 53(12), 3048-3056. DOI:10.2337/ diabetes.53.12.3048.

Lê, K. A., Faeh, D., Stettler, R., Ith, M., Kreis, R., Vermathen, P., Boesch, C., Ravussin, E. \& Tappy, L. (2006). A 4-wk high-fructose diet alters lipid metabolism without affecting insulin sensitivity or ectopic lipids in healthy humans. The American Journal of Clinical Nutrition, 84(6), 13741379. DOI:10.1093/ajcn/84.6.1374.

Lindqvist, A., Baelemans, A. \& Erlanson-Albertsson, C. (2008). Effects of sucrose, glucose and fructose on peripheral and central appetite signals. Regulatory Peptides, 150(1-3), 26-32. DOI: 10.1016/j.regpep.2008.06.0.

Liu, H., Huang, D., McArthur, D. L., Boros, L. G., Nissen, N. \& Heaney, A. P. (2010). Fructose induces transketolase flux to promote pancreatic cancer growth. Cancer Research, 70(15), 6368-6376. DOI: 10.1158/0008-5472.can-09-4615.

Mayes, P. A. (1993). Intermediary metabolism of fructose. The American Journal of Clinical Nutrition, 58(5), 754S-765S. DOI: $10.1093 /$ ajen/58.5.754S.

Nöthlings, U., Murphy, S. P., Wilkens, L. R., Henderson, B. E. \& Kolonel, L. N. (2007). Dietary glycemic load, added sugars, and carbohydrates as risk factors for pancreatic cancer: The multiethnic cohort study. The American Journal of Clinical Nutrition, 86(5), 1495-1501. DOI:10.1093/ajcn/86.5.1495.

Olivares-Reyes, J. A. \& Arellano-Plancarte, A. (2008). Bases moleculares de las acciones de la insulina. Revista de Educación Bioquímica, 27(1), 9-18.

Ozawa, T., Maehara, N., Kai, T., Arai, S. \& Miyazaki, T. (2016). Dietary fructose-induced hepatocellular carcinoma development manifested in mice lacking apoptosis inhibitor of macrophage (AIM). Genes Cells, 21(12), 1320-1332. DOI: $10.1111 /$ gtc. 12446 .

Pektaş, M. B., Sadi, G. \& Akar, F. (2015). Long-term dietary fructose causes gender-different metabolic and vascular dysfunction in rats: Modulatory effects of resveratrol. Cellular Physiology and Biochemistry, 37(4), 1407-
1420. DOI:10.1159/000430405.

Robubi, A., Huber, K. R. \& Krugluger, W. (2014). Extra fructose in the growth medium fuels lipogenesis of adipocytes. Journal of Obesity, 2014(1), 1-6. DOI: 10.1155/2014/647034.

Roglans, N., Vila, L., Farre, M., Alegret, M., Sánchez, R. M., Vazquez-Carrera, M. \& Laguna J. C. (2007). Impairment of hepatic Stat-3 activation and reduction of PPARalpha activity in fructose-fed rats, Hepatology, 45(3), 778-788. DOI: $10.1002 /$ help. 21499 .

Rutledge, A. C. \& Adeli, K. (2008). Fructose and the metabolic syndrome: Pathophysiology and molecular mechanisms. Nutrition Reviews, 65(6), 13-23. DOI: 10.1111/j.17534887. 2007.tb00322.x.

Samuel, V. T., Liu, Z. X., Qu, X., Elder, B. D., Bilz, S., Befroy, D., Romanelli, A. J. \& Shulman, G. I. (2004). Mechanism of hepatic insulin resistance in non-alcoholic fatty liver disease. Journal of Biological Chemistry, 279(31), 32345 32353. DOI: 10.1074/jbc.m313478200.

Shu, H. J., Isenberg, K., Cormier, R. J., Benz,A. \& Zorumski, C. F. (2006). Expression of fructose sensitive glucose transporter in the brains of fructose-fed rats. Neuroscience, 140(3), 889-895. DOI: 10.1016/j.neuroscience.2006.02.071.

Shulman, G. I. (2000). Cellular mechanisms of insulin resistance. Journal of Clinical Investigation, 106(2), 171-176. DOI: 10.1172/jci10583.

Soria, J, (2010) Análisis causa y efecto de la ingesta de jarabe de alta fructosa (HFSC). [Tesis de licenciatura]. 1-40. Ciudad de México. Instituto Politécnico Nacional.

Spruss, A., Kanuri, G., Wagnerberger, S., Haub, S., Bischoff, S. C. \& Bergheim, I. (2009). Toll-like receptor 4 is involved in the development of fructose-induced hepatic steatosis in mice. Hepatology, 50(4), 1094-1104. DOI:10.1002/ hep. 23122.

Stanhope, K. L., Schwarz, J. M., Keim, N. L., Griffen, S. C., Bremer, A. A., Graham, J. L., Hatcher, B., Cox, C.L., Dyachenko, A., Zhang, W., McGahan, J. P., Seibert, A., Krauss, R. M., Chiu, S., Schaefer, E. J., Ai, M., Otokozawa, S., Nakajima, K., Nakano, T., Beysen, C., Hellerstein, M. K., Berglund, L. \& Havel, P. J. (2009). Consuming fructose-sweetened, not glucosa-sweetened, beverages increases visceral adiposity and lipids and decreases insulin sensitivity in verweight/obese humans. The Journal of Clinical Investigation, 119(5), 1322-1334. DOI: 10.1172/ JCI37385.

Strober, J. W. \& Brady, M. J. (2019). Dietary fructose consumption and triple-negative breast cancer incidence. Frontiers in Endocrinology, 12(10), 1-7. DOI: 10.3389/ fendo.2019.00367.

Szendroedi, J., Yoshimura, T., Phielix, E., Koliaki, C., Marcucci, M., Zhang, D., Jelenik, T., Müller, J., Herder, C., Nowotny, P., Shulman, G. I. \& Roden, M. (2014). Role of diacylglycerol activation of $\mathrm{PKC} \theta$ in lipid-induced muscle insulin resistance in humans. Proceedings of the National 
Academy of Sciences, 111(26), 9597-9602. DOI: 10.1073/ pnas. 1409229111.

Tappy, L. \& Lê K. A. (2010). Metabolic effects of fructose and the worldwide increase in obesity. Physiolical Reviews, 90(1), 23-46. DOI: 10.1152/physrev.00019.2009.

Theytaz, F., de Giorgi, S., Hodson, L., Stefanoni, N., Rey, V., Schneiter, P., Giusti, V. \& Tappy, L. (2014). Metabolic fate of fructose ingested with and without glucose in a mixed meal. Nutrients, 6(7), 2632-2649. DOI :10.3390/ nu6072632.

Tsai, J., Zhang, R., Qiu, W., Su, Q., Naples, M. \& Adeli, K. (2009). Inflammatory $\mathrm{NF}-\kappa \mathrm{B}$ activation promotes hepatic apolipoprotein B100 secretion: evidence for a link between hepatic inflammation and lipoprotein production. American Journal of Physiology-Gastrointestinal and Liver Physiology, 296(6), G1287-G1298. DOI:10.1152/ ajpgi.90540.2008.

Veech, R. L., (2003). A humble hexose monophosphate pathway metabolite regulates short- and long-term control of lipogénesis. Proceedings of the National Academy of Sciences, 100(10), 5578-5580. DOI:10.1073/ pnas. 1132039100.

Zavaroni, I., Sander, S., Scott, S. \& Reaven, G. M. (1980). Effect of fructose feeding on insulin secretion and insulin action in the rat. Metabolism, 29(10), 970-973. DOI:10.1016/00260495(80)90041-4. 